RESEARCH REPORT

\title{
THE EFFECTS OF MUSCLE ENERGY TECHNIQUE AND STATIC STRETCHING OF GASTROCNEMIUS ON PAIN AND DISABILITY IN KNEE OSTEOARTHRITIS
}

\begin{abstract}
BACKGROUND AND AIMS

Knee Osteoarthritis (OA) is a degenerative disorder and a leading cause of disability associated with pain, restricted ROM and difficulty in performing Activities of Daily Living (ADLs). The aim of this study was to compare the effectiveness of Muscle Energy Technique (MET) and static stretching on gastrocnemius muscles among the patients suffering with knee $O A$.
\end{abstract}

\section{METHODOLOGY}

A 2-arm Randomized Control Trial was conducted at Ziauddin Hospital among 120 patients suffering with grade-II OA. Initial screening was conducted on the basis of Knee OOS. Group A received the MET whereas Group B received Static Stretching Technique both group received the treatment for three days/week for 4 weeks.

\section{RESULTS}

In initial evaluation, our results revealed that group A shows the KOOS score $0.43 \pm 0.10$ that improved significantly after four weeks of treatment to $0.49 \pm 0.14$. Our results revealed that at $95 \% \mathrm{Cl}$, the KOOS score of patients significantly improved in both the groups $p<0.05$

\section{CONCLUSION}

Our study concluded that Muscle Energy Technique is found to be more effective technique in reducing pain and functional disability in knee OA patients.

\section{KEYWORDS}

Pain, Degenerative, Osteoarthritis, Symptoms, Limitation of Activity, Rehabilitation

\author{
Amna Aamir Khan \\ Assistant Professor \\ Ziauddin College of Rehabilitation Sciences \\ Ziauddin University \\ akhan39@caledonian.ac.uk \\ Jaza Rizvi \\ Lecturer \\ Ziauddin College of Rehabilitation Sciences \\ Ziauddin University \\ jaza.rizvi@zu.edu.pk
}

[Khan AA, Rizvi J. The Effects of MET And Static Stretching of Gastrocnemius on Pain and Disability in Knee Osteoarthritis. Pak. j. rehabil. 2018;7(2)32-37] 


\section{INTRODUCTION}

Knee Osteoarthritis (OA) is considered as the leading cause of disability between both men and women. The disease associated with pain, restricted range of motion and deformity leading to difficulty in performing Activities of Daily Living (ADLS) and poor quality of life $e^{1-2}$. With the increase in life expectancy, the prevalence of OA is $28 \%$ in rural and $25 \%$ in urban areas ${ }^{3}$. However, OA is incurable therefore the aim of management of knee $\mathrm{OA}$ is to conserve the function and enhance quality of life'. Therefore, the classical presentation of OA occurs with limited range of motion of knee joint leading to swelling, loss of quadriceps strength and formation of bony spurs $^{4}$. Therefore, functional impairment is lost due to failure of voluntary activation ${ }^{5}$. In addition, the biomechanical changes with respect to pathophysiological consequences of OA cause alterations in articular cartilage and surrounding ligaments thereby increases the joint compression and load ${ }^{6}$. Furthermore, lack of quadriceps and hamstrings co-activation in OA leads to greater instability among women ${ }^{7}$. Additional factors associated with OA are gender, family history, overuse of joints, obesity etc. Among all, obesity is more prevalent in degenerative diseases as the pressure on knee increases whereas weight is shifted downwards which directly affects the articular cartilage ${ }^{8}$.

Number of studies reported the benefits of manual therapy techniques used in combination with joint mobility and strengthening exercises in order to improve functional performance and decrease symptoms 9 . Similarly, several exercise training programs in strengthening of quadriceps and hamstrings have been documented whereas limited evidence is available on training protocols for strengthening of gastrocnemius muscles. Amidst variety of techniques available, one of the renowned and advance stretching manual technique named as Muscle Energy Technique (MET) has been distinguished for releasing the contractures, stretching of muscles and increasing the $\mathrm{ROM}^{10}$. On the contrary, static stretching may be considered as a preferable method to enhance flexibility and joint ROM due to its feasibility ${ }^{11}$. However, inadequate evidence is available on static stretching to prevent risk of musculoskeletal disorders and improving overall flexibility and restricted joint ROM.

Another study conducted to determine impact of MET to improve hamstrings flexibility among collegiate males ${ }^{12}$. Outcomes of the study concluded that MET not only increases the muscle length but also increases the tolerance to stretch by biomechanical and neurophysiological changes to connective tissue. As MET prompts these mechanisms therefore the underlying changes addresses to both ROM and muscle stiffness following the application of the technique.

Number of studies also reported the significance of post isometric relaxation technique in hamstrings flexibility which increases ROM and reduces muscle spasm $^{13}$. Moreover, strong evidence derived from the recent researches concluded that MET has great influence on tight muscles as it releases the muscles at faster rate than any other technique and ultimately increases the joint range of motion and muscle flexibility ${ }^{14}$.

Multiple trials using MET or stretching technique has been conducted on neck pain which concluded that both techniques are individually beneficial in management of mechanical neck pain ${ }^{15}$. However, when compared, MET seem to be more effective than stretching in reducing pain and functional disability. Whereas, limited evidence is found on using MET in patients with musculoskeletal disorders like OA, tendinitis, fibromyalgia etc.

In addition, MET can be used as an adjunct therapy in reducing pain and functional disability in various pain categories. Furthermore, the finding of recent studies suggests that exercise-based physiotherapy interventions may reduce the prerequisite for surgeries or other treatments like knee arthroplasty, intra-articular injections etc. Yet, the efficacy of combinations of exercises and their dosage is still unclear $^{16}$. In order to provide exercise based physiotherapy intervention, this study aims to intervene the MET and stretching exercise particularly on gastrocnemius muscles to evaluate the resulting changes in pain and disability.

\section{METHODOLOGY}

\section{Study Setting}

The study was conducted at the outpatient department of rehabilitation sciences Dr. Ziauddin Hospital in all three campuses that were Kemari, Clifton and North Nazimabad.

\section{Target Population}

The patients with knee osteoarthritis grade-II were enrolled in the study

\section{Study Design}

A single blind, two-arm Randomized Controlled Trial was conducted

\section{Duration of Study}

8 months.

\section{Sample Technique}

A simple random sampling technique was done through toss a coin method here patients receiving heads were enrolled in group A whereas all the tails were sent in group B.

\section{Inclusion/Exclusion Criteria}

Patients aged 40-60 years including both genders suffering with grade II osteoarthritis were selected for the study ${ }^{17}$. Patients with surgery within six months $(6 \text { months })^{18}$, systemic bone diseases ${ }^{17}$, total knee replacement ${ }^{19}$ and with intra-articular injections 
were excluded from the study ${ }^{20}$.

\section{Data Collection Procedure}

The patients were recruited from the Ziauddin hospital that came in out-patient physical therapy department. Initially, patient was assessed according to inclusion criteria, and the procedure was explained prior. A written consent form was taken from the patient assuring their voluntary participation. One hundred and twenty patients were randomly and equally divided into two subgroups where Group A received the Muscle Energy Technique and Group B received static stretching technique. Patients received intervention on every alternative day in a week for 3 days per week for 4 weeks. The treatment was given under the supervision of trained senior Physical therapist. Patients were assessed for Knee related disability via KOOS scale (Knee Injury and Osteoarthritis Outcome Score) at the start of the first session where pain, symptoms, activities of daily living (ADL), function in Sport and Recreational activities and knee-related quality of life (QOL) were documented. This scale was based on Likert comprising of never, rarely, sometimes, often and always criteria. Symptoms comprises of seven questions, pain nine, ADLs seventeen, sports five and quality of life four. The pain was reported on the frequency, intensity and its associated factors. Moreover, the symptoms and stiffness were documented based on the severity of osteoarthritis. For ADLs stair climbing, sitting, standing, bending, walking, driving, shopping, clothing, toileting, bathing, lying and domestic duties were considered. For sports squatting, running, jumping, twisting, turning and kneeling were documented. Quality of life consisted of awareness, life style modification, lack of confidence and difficulties were reported. Good to excellent reliability and validity of KOOS has already been documented (Cronbach's alpha: pain 0.91; symptoms 0.75; ADLs 0.96; function in sports and recreation 0.86 and knee-related Quality of Life is 0.74). Patient was reassessed after the end of 6th session and at the end of 12th session in a same manner.

\section{Treatment Protocol for Group A (MET)}

Post Isometric Relaxation (PIR) protocol was given in MET training. Initially, hot pack was applied for 10 minutes to increase the blood supply while the patient was supine lying on a comfortable plinth with leg straight, therapist holding the ankle with his/her hand. Then patient was asked to perform planter flexion with resistance provided by the therapist in a way that there was no movement at ankle joint (isometric contraction). Patient was advised to hold it for 7-10 seconds (while pushing the therapist hand patient applied only mild force. After it therapist stretched the gastrocnemius by its full available pain free range and hold it for 10 seconds. This procedure was repeated 4-5 times per session ${ }^{18}$.

\section{Treatment Protocol for Group B (Static Stretching)}

Patient lie supine on a comfortable plinth with straight leg, hot pack applied over the calf muscles for 10 minutes before initiating the static stretching technique to increase the blood supply over the muscles. Therapist performed the dorsiflexion and hold it for 30 seconds followed by 15-20 seconds rest, 20 stretches were performed in each session 21 .

\section{DATA ANALYSIS}

Data was analyzed and interpreted on SPSS (Statistical Package for Social Sciences) version 22.0. Descriptive statistics was performed for participants' demographic characteristics. Paired T-test was applied for within the group analysis while independent T-test was executed for between the group post mean differences whereas, significant P-value $<0.05$ was considered for both the groups.

\section{RESULT}

Total 120 participants, 60 in each group respectively completed the protocol with mean age of $50.7 \pm 5.59$ in Group A with 30 male and female subjects correspondingly whereas in Group B, 35 participants were male and 25 were females with mean age of $51.51 \pm 6.25$. Participants were assessed on KOOS at 4 weeks pre and post intervention; moreover no loss of attrition has been reported. Baseline Characteristics of participants are shown in Table-1.

Within group analysis was performed using paired T-test at $95 \%$ of $\mathrm{Cl}$ and critical value $\mathrm{f} 5 \%$. The results revealed that pre-post analysis of $5 \%$ of alpha was found to be significant $\mathrm{p}<0.05$ in both the groups from baseline till four weeks. The total pre KOOS

Table 1. Shows the baseline characteristics of patients suffering with Knee Osteoarthritis

\begin{tabular}{|c|c|c|c|c|c|c|c|c|c|}
\hline & $\begin{array}{c}\text { Age } \\
\text { (Years) }\end{array}$ & \multicolumn{2}{|c|}{ Gender } & \multicolumn{6}{|c|}{ KOOS } \\
\hline & & Male & Female & Pain & Symptoms & ADLs & $\begin{array}{l}\text { Sports \& } \\
\text { Recreation }\end{array}$ & QoL & Total \\
\hline MET Mean \pm S.D & $50.7 \pm 5.5$ & 30 & 30 & $0.48 \pm 0.1$ & $0.50 \pm 0.1$ & $0.50 \pm 0.1$ & $0.35 \pm 0.1$ & $0.40 \pm 0.1$ & $0.43 \pm 0.1$ \\
\hline $\begin{array}{l}\text { Stretching } \\
\text { Mean } \pm \text { S.D }\end{array}$ & $51.51 \pm 6.2$ & 35 & 25 & $0.54 \pm 0.1$ & $0.58 \pm 0.2$ & $0.55 \pm 0.1$ & $0.09 \pm 0.1$ & $0.44 \pm 0.1$ & $0.49 \pm 0.1$ \\
\hline
\end{tabular}


mean after MET training improved from $0.43 \pm 0.10$ to $0.60 \pm 0.90$ after 4 weeks whereas in stretching group it improved from $0.49 \pm 0.14$ to $0.58 \pm 0.16$. Therefore an overall mean difference of 0.16 and 0.08 was found respectively as shown in Table-2. was found to be equally effective in reducing pain and symptoms of osteoarthritis but showed no significant improvement in ADLs. Hence the technique is least likely to be effective then MET. Number of studies have supported MET as an effective

\begin{tabular}{|c|c|c|c|c|}
\hline & $\begin{array}{c}\text { Pre KOOS } \\
\text { Mean士S.D. }\end{array}$ & $\begin{array}{l}\text { Post KOOS } \\
\text { Mean士S.D. }\end{array}$ & $\begin{array}{c}\text { Mean } \\
\text { Difference }\end{array}$ & P-value \\
\hline MET & $0.43 \pm 0.10$ & $0.60 \pm 0.90$ & 0.16 & $<0.05$ \\
\hline Stretching & $0.49 \pm 0.14$ & $0.58 \pm 0.16$ & 0.08 & $<0.05$ \\
\hline
\end{tabular}

The change in the KOOS sub-scales was determined through Independent T-test after 4 weeks of training. The difference between the two groups was analyzed using post mean values of components. In general, Group A reported significant improvement $(p<0.05)$ in comparison to Group B with mean value of $0.62 \pm 0.01$ on total KOOS post the intervention as shown in Table-3 treatment protocol. In particular, a study conducted by Abdelkarim ${ }^{22}$ observed the viability of static stretching and MET on hamstrings among patients with post-burn contractures. The outcomes of the study inferred that MET proved to be beneficial than static stretching in the duration of eight days of treatment as MET prompt the relaxation of hypotonic musculature and consequent extending of the

Table 3. Shows the Comparison of KOOS sub-scales between the groups
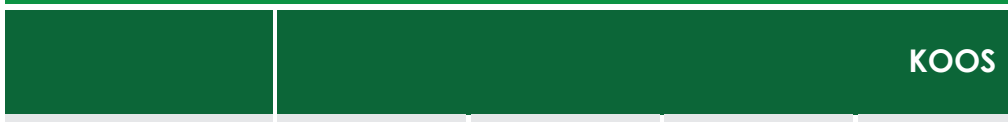

\section{KOOS}

\begin{tabular}{|c|c|c|c|c|c|c|c|}
\hline & Pain & Symptoms & ADLs & $\begin{array}{c}\text { Sports \& } \\
\text { Recreation }\end{array}$ & QoL & Total & P-Value \\
\hline $\begin{array}{c}\text { MET } \\
\text { (Change In } \\
\text { KOOS Scores) }\end{array}$ & $0.66 \pm 0.1$ & $0.66 \pm 0.1$ & $0.70 \pm 0.1$ & $0.55 \pm 0.12$ & $0.60 \pm 0.1$ & $0.60 \pm 0.0$ & \multirow{2}{*}{$<0.05^{*}$} \\
\hline $\begin{array}{c}\text { Stretching } \\
\text { (Change In } \\
\text { KOOS Scores) }\end{array}$ & $0.62 \pm 0.1$ & $0.65 \pm 0.2$ & $0.65 \pm 0.1$ & $0.14 \pm 0.25$ & $0.54 \pm 0.1$ & $0.58 \pm 0.1$ & \\
\hline
\end{tabular}

*Significant P-value for between the group differences at 4 weeks

Interestingly, KOOS ADLs of Group A showed marked increase of $0.70 \pm 0.10$ as compared to Group B with $0.65 \pm 0.19$ mean value. Similarly for Group A, Sports and Recreation values increases $0.55 \pm 0.12$, whereas Group B showed no difference with mean of $0.14 \pm 0.25$ respectively. Furthermore, QoL in group A also increased after receiving 4 weeks of treatment $0.60 \pm 0.13$ where moderate improvement was reported in Group B with $0.54 \pm 0.15$ post mean values when compared to baseline. In comparison, sub-scales of pain and symptoms showed significant improvement in both the groups as represented in Table-3

\section{DISCUSSION}

Our study shows that the group receiving MET treatment has marked improvement on KOOS subscalewhen compared with stretching group. Therefore, increasing the quality of life and decreasing the functional disability. However, stretching method muscle in comparison to static stretching. On the contrary, Kostopolouset $a^{23}$ shows a massive pain decrease within the group when applying stretching of upper trapezius, yet the impact is still unknown.

However, the impact of conventional treatment cannot be ignored that comprises of application of heat, stretching and strengthening exercises. As multiple studies has shown significant results and is routinely practiced in the clinical settings. In addition, Brakke and Schencking ${ }^{24}$ reported impact of TENS electrical stimulation on knee OA patients in comparison to other treatment approaches. Results of the study reported that stretching exercises have better outcomes than any other modalities in conventional rehabilitation of $O A$.

Although Shenouda ${ }^{25}$ shows that stretching and MET are similarly effective protocols for improving the ROM and decreasing pain. Similar results were obtained in our study when pain was markedly reduced in MET group $0.66 \pm 0.10$ whereas for stretch- 
ing it was $0.62 \pm 0.18$. This was further endorsed in a study conducted by Paremeret $\mathrm{al}^{26}$. Despite the fact that MET was successful in several studies, the assessment parameters with respect to patient's functional disability did not fulfill the long term effects of interventions. Moreover, Robertset $\mathrm{a}^{27}$ indicated the short-term effects of MET to decrease pain, muscle tension, spasm and increase strength. Therefore the effects in the study lasted from a few seconds to minutes, but if continued MET protocol is applied then these effects may last for longer duration. Researchers today have been unclear regarding the response of a single MET session.

Interestingly, the results obtained in the MET group with respect to activities of daily living and quality of life showed significant results in the within group

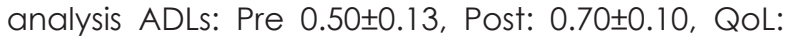
Pre:0.40 \pm 0.16 , Post $0.60 \pm 0.13$. These results were consistent with the study conducted by Sharmila ${ }^{28}$ et al on MET where it was reported as favorable treatment approach. Although MET and stretching are broadly used within the discipline of physical therapy but research usage of these two strategies are limited to symptomatic population.

Interestingly, static stretching had also shown to be effective to enhance ROM of knee joint as Day et $\mathrm{al}^{29}$ shows 30 minutes of stretching increased in fascicle length of the soleus and lateral gastrocnemius about eight millimeter(8-mm). Moreover, Maguusonet $\mathrm{al}^{30}$ have also suggested a 4 weeks of static stretch to improve the flexibility. Usually stretching exercises helps in increasing the ROM that ultimately impacts the athletic performance. The KOOS sports and recreational score in the stretching group also showed improvements Pre: $0.09 \pm 0.15$, post $0.14 \pm 0.25$. Our results were statistically significant $(p<0.05)$ but clinically non-significant. As noticed in our study, MET was successful in reducing pain and symptoms, enhancing the ADLs, QoL and Sports and recreational activities in comparison with stretching of knee OA within the treatment in knee OA pain. The overall impact of MET on the KOOS scale was higher when compared with the stretching group (MET Post: 0.60 \pm 0.09 ; Stretching Post $0.58 \pm 0.16)$ with both statistically and clinically significant results $(\mathrm{p}<0.05)$.

Consequently, KOOS outcome scores were significant to evaluate long term status of the patients therefore it provided a better estimation on overall quality of life, daily living activities and associated pain or symptoms of the disease. It was observed in a 1-year follow-up study in which participants' pain was evaluated on Visual Analogue Scale (VAS) during the knee joint movement. The parameter was assessed on Pain items in KOOS questionnaire in patients who performed exercises showed better results than the control group ${ }^{31}$. Activities on KOOS scale represented improved ability of the patients in activities such as walking and bathing. Consecutively, the questionnaire also reported the gradual reduction of pain and symptoms related to the severity of disease whereas evaluates the long-term status after recommencing of ADL's and sports activities.

\section{CONCLUSION}

Our study concluded that Muscle Energy Technique is found to be more effective rehabilitation technique in reducing pain and functional disability in knee OA patients. Also, MET has shown to be a markedly beneficial technique in improving Activity of Daily Living and Quality of Life on Knee injury and Osteoarthritis Outcome Score. Although the traditional technique that is static stretching technique has also shown significant improvement in decreasing pain and symptoms of the disorders on KOOS sub-scales but larger scale studies are to be conducted considering all the grades of osteoarthritis separately and comparing the results of improvements. Moreover, other conservative treatment therapies should also be documented in future studies.

\section{REFERENCES}

[1] Aoki O, Tsumura N, Kimura A, Okuyama S, Takikawa S, Hirata S. Home stretching exercise is effective for improving knee range of motion and gait in patients with knee osteoarthritis.JPhysTher Sc. 2009:21 (2):113-9.

[2] Neogi T. The epidemiology and impact of pain in osteoarthritis. Osteoarthritis and Cartilage. 2013;21 (9):1 145-53.

[3] labal MN, Haidri FR, Motiani B, Mannan A. Frequency of factors associated with knee osteoarthritis. J Pak Med Assoc. $2011 ; 61$ (8):786.

[4] Lespasio MJ, Piuzzi NS, Husni ME, Muschler GF, Guarino AJ, Mont MA. Knee osteoarthritis: a primer. Perm J.2017;21.

[5] Alnahdi AH, Zeni JA, Snyder-Mackler L. Muscle impairments in patients with knee osteoarthritis. Sports Health. 2012 (4):284-92.

[6] Heijink A, Gomoll AH, Madry $H$, Drobnič $M$, Filardo G, Espregueira-Mendes J, Van Dijk CN. Biomechanical considerations in the pathogenesis of osteoarthritis of the knee. Knee Surgery, Sports Traumatology, Arthroscopy. 2012 ;20(3):423-35

[7] Thomas AC, Judd DL, Davidson BS, Eckhoff DG, Stevens-Lapsley JE. Quadriceps/hamstrings co-activation increases early after total knee arthroplasty. The Knee. $2014 ; 21$ (6):1115-9.

[8] Sowers $M$, Karvonen-Gutierrez CA, Palmieri-Smith R, Jacobson JA, Jiang $Y$, Ashton-Miller JA. Knee osteoarthritis in obese women with cardiometabolic clustering. Arthritis Care \&

[9] Research. 2009;61 (10):1328-36.

Golightly YM, Allen KD, Caine DJ. A comprehensive review of the effectiveness of different exercise programs for patients with osteoarthritis. The Physician and sportsmedicine. 
2012;40(4):52-65.

[10] Phadke A, Bedekar N, Shyam A, Sancheti P. Effect of muscle energy technique and static stretching on pain and functional disability in patients with mechanical neck pain: A randomized controlled trial.

Hong Kong Physiother J. $2016 ; 35: 5-11$.

[11] Shoukat H, Arshad HS, Sharif F, Fatima A, Shoukat $F$. Effects of different stretching times on range of motion in patients with hamstring tightness: A randomised control trial. Annals of King Edward Medical University. 2017;23(4):554-9.

[12] Waseem M, Nuhmani S, Ram CS. Efficacy of Muscle Energy Technique on hamstring muscles flexibility in normal Indian collegiate males. Calicut medical journal. 2009;7(2):e4.

[13] Lewit K. Postisometric relaxation in combination with other methods of muscular facilitation and inhibition. Manual Medicine. 1986;2:101-4.

[14] Page P. Current concepts in muscle stretching for exercise and rehabilitation.Int J Sports PhysTher. 2012 (1):109.

[15] Mahajan R, Kataria C, Bansal K. Comparative effectiveness of muscle energy technique and static stretching for treatment of subacute mechanical neck pain. Int J Health Rehabil Sci. 2012;1 (1):16-21.

[16] Deyle GD, Allison SC, Matekel RL, Ryder MG, Stang JM, Gohdes DD, Hutton JP, Henderson NE, Garber MB. Physical therapy treatment effectiveness for osteoarthritis of the knee: a randomized comparison of supervised clinical exercise and manual therapy procedures versus a home exercise program. Physical therapy. 2005 ;85(12):1301-17.

[17] Khalid MU, Akhtar MA, Akhtar MH. Frequency of osteoarthritis among patients of knee joint pain. JSZMC. 2015;6(4):885-7.

[18] Shadmehr A, Hadian MR, Naiemi SS, Jalaie S. Hamstring flexibility in young women following passive stretch and muscle energy technique. J Back MusculoskeletRehabil. 2009;22(3):143-8.

[19] Knoop J, Dekker J, Van Der Leeden M, Van der Esch M, Thorstensson CA, Gerritsen M, Voorneman RE, Peter WF, de Rooij M, Romviel S, Lems WF. Knee joint stabilization therapy in patients with osteoarthritis of the knee: a randomized, controlled trial. Osteoarthritis and cartilage. 2013;21 (8):1025-34.

[20] Ali SS, Ahmed SI, Khan M, Soomro RR. Comparing the effects of manual therapy versus electro physical agents in the management of knee osteoarthritis. Pak J Pharm Sci. 2014; 27/4 Suppl):1103-6.

[21] Babault N, Kouassi BY, Desbrosses K. Acute effects of 15 min static or contract-relax stretching modalities on plantar flexors neuromuscular properties. J Sci Med Sport. 2010;13(2):247-52.

[22] Ahmed ET, Abdelkarim SS. Efficacy of muscle energy technique versus static stretching technique in increasing hamstring flexibility post burn contracture. Int J Health Rehabil Sci. 2013;2(1):22-7.

[23] Kostopoulos D, Nelson Jr AJ, Ingber RS, Larkin RW. Reduction of spontaneous electrical activity and pain perception of trigger points in the upper trapezius muscle through trigger point compression and passive stretching. Journal of Musculoskeletal Pain. $2008 ; 16(4): 266-78$.

[24] Nejati P, Farzinmehr A, Moradi-Lakeh M. The effect of exercise therapy on knee osteoarthritis: a randomized clinical trial. Med J Islam Repub Iran. 2015;29:186.

[25] Shenouda MM. Efficacy of Stretching Exercises VersusPostisometric Relaxation Technique on Pain, Functional Disability and Range of Motion in Patients with Cervical Spondylosis. A Randomized controlled trial. Bulletin of Faculty of Physical Therapy. 2012;17(2).

[26] Parmar S, Shyam A, Sabnis S, Sancheti P. The effect of isolytic contraction and passive manual stretching on pain and knee range of motion after hip surgery: A prospective, double-blinded, randomized study.

[27] Hong Kong Physiother J. 2011 ;29(1):25-30.

Roberts BL. Soft tissue manipulation: neuromuscular and muscle energy techniques.JNeurosciNurs. 1997;29(2):123-8.

[28] Sharmila B. Isometric Muscle Energy Technique and Non-Specific Neck Pain in Secondary School Teachers-Results of an Experimental Study. Indian J PhysiotherOccupTher. 2014 ;8(2):58.

[29] Day JM, Nitz AJ. The effect of muscle energy techniques on disability and pain scores in individuals with low back pain. J Sport Rehabil. $2012 ; 21(2): 194-8$

[30] Babault N, Kouassi BY, Desbrosses K. Acute effects of 15 min static or contract-relax stretching modalities on plantar flexors neuromuscular propertiesJSci Med Sport. 2010 1;13(2):247-52.

[31] Wasserstein D, Huston LJ, Nwosu S, Kaeding CC Parker RD, Wright RW, Andrish JT, Marx RG, Amendola A, Wolf BR, McCarty EC. KOOS pain as a marker for significant knee pain two and six years after primary $A C L$ reconstruction: a Multicenter Orthopaedic Outcomes Network (MOON) prospective longitudinal cohort study. Osteoarthritis and cartilage. 2015;23(10):1674-84. 